\title{
InterCriteria Analysis of data from intellectual and physical evaluation tests of students practicing sports activities
}

\author{
Simeon Ribagin ${ }^{1,2}$ and Spas Stavrev ${ }^{3}$ \\ ${ }^{1}$ Bioinformatics and Mathematical Modelling Department, \\ Institute of Biophysics and Biomedical Engineering, Bulgarian Academy of Sciences \\ 105 Acad. Georgi Bonchev Str., Sofia 1113, Bulgaria \\ 2 Department of Health and Social Care, Medical College, \\ University "Prof. D-r Asen Zlatarov", Burgas 8010, Bulgaria \\ e-mail: simribagin@gmail. com \\ ${ }^{3}$ Physical Culture and Sport Department, University of National and World Economy \\ Students Town, Sofia 1700, Bulgaria \\ e-mail: stavrevspas@unwe.bg
}

\begin{abstract}
Physical activity among university students is essential for complementing sedentary behaviour and for individuals' future mental and physical health. At the same time, intellectual qualities like operative, analytic, logical thinking, attention span, short-term memory, long-term memory, etc., are important factors for the successful participation in different academic sports activities. In view of this, it is important to evaluate the initial level of intellectual and physical development by applying a group of specific tests. In this paper, we propose the application of the approach of InterCriteria Analysis (ICrA) to data, obtained from university students practicing sports activities in order to evaluate the appropriateness of the used tests.
\end{abstract}

Keywords: Physical activity, University students, InterCriteria Analysis, Intuitionistic fuzzy sets. 2010 Mathematics Subject Classification: 03 E72.

\section{Introduction}

Physical condition and intellectual development monitoring is important for assessing healthrelated aspects during the life span. It is important to monitor trends in health and physical activity patterns in early adult life as this represents a period in which health behaviors get established and physical inactivity can manifest $[11,8]$. The beginning of the university stage is usually 
accompanied by physiological and psychological changes associated with late adolescence and emerging adulthood [9]. It should also be noted that it is an important stage of life with great changes in behaviours and responsibilities derived from emancipation and the demands developed by a new university degree [6]. Changes in the levels of physical activity during university studies may reflect disruptions in established living patterns that persist into the workforce [7]. Moreover, the promotion of university student health has been shown to enhance future wellbeing and career performance [5]. In view of this, it is important to evaluate the initial level of intellectual and physical development by applying a group of specific tests. Nevertheless, it is of great importance to evaluate the usefulness of the applied method of testing and to establish a well-structured test battery. In this paper, we propose the application of the approach of InterCriteria Analysis (ICrA) to data, obtained from university students practicing sports activities. The data was analyzed in search of correlations between the results from the method of testing for the level of development of the motive qualities, as well as tests for assessment of the psychic and personality qualities in order to predict the reliability of the proposed test battery.

\section{Presentation of the input data}

The investigation and the data collection process, has been carried out in the educational year 2011/12. Contingent of the investigation are 66, 1st year students (males) in UNWE, attending basketball and table tennis trainings from "Physical Culture" subject. We have chosen a set of several parameters defining the level of cognitive and emotional well-being in a test battery of 13 control tasks for intellectual status, and 5 control tasks for physical condition. These tests have been divided into two major groups, as follows:

\section{Physical condition}

- Endurance. It is the ability of an organism to exert itself and remain active for a long period of time (C1),

- Grip strength. It is the force applied by the hand to pull on or suspend from objects and is a specific part of hand strength (C2),

- Agility. It is the ability to change the body's position efficiently, and requires the integration of isolated movement skills (C3),

- Balance. It is an ability to maintain the line of gravity (vertical line from center of mass) of a body within the base of support with minimal postural sway (C4),

- Skillfulness. It is the ability to perform different tasks well (C5).

\section{Personality}

- Operational thinking. This type of thinking repeats and illustrates action, preceding or following it within a limited temporal span (C6),

- Analytical thinking. This type is a critical component of visual thinking that gives one the ability to solve problems quickly and effectively (C7),

- Logical thinking. This is the process in which one uses reasoning consistently to come to a conclusion (C8),

- Visual memory. It is a form of memory which preserves some characteristics of our senses pertaining to visual experience (C9 and $\mathrm{C} 10)$,

- Willpower. This is the cognitive process by which an individual decides on and commits to a particular course of action (C11), 
- Personal activity. The person's skill to participate actively in different activities (C12),

- Communication skills. These allows you to understand and be understood by others (C13),

- Organizational skills. Refers to our ability to stay focused on different tasks, and use your time, energy, strength, mental capacity, physical space, etc. effectively and efficiently in order to achieve the desired outcome (C14),

- Morality. It is the differentiation of intentions, decisions and actions between those that are distinguished as proper and those that are improper (C15).

- Neuroticism. It is one of the Big Five higher-order personality traits in the study of psychology. Individuals who score high on neuroticism are more likely than average to be moody and to experience such feelings as anxiety, worry, fear (C16),

- Extraversion and Introversion. Extraversion tends to be manifested in outgoing, talkative, energetic behavior, whereas introversion is manifested in more reserved and solitary behavior (C17),

- Anxiety. It is a normal emotion that causes increased alertness, fear, and physical signs, such as a rapid heart rate $(\mathrm{C} 18)$,

The results from the tests, as an input data are obtained from two control groups of students and are listed below in Table 1 and Table 2.

\begin{tabular}{|c|c|c|c|c|c|c|c|c|c|c|c|c|c|c|c|c|c|c|}
\hline & C1 & 2 & C3 & $\mathrm{C4}$ & C5 & C6 & C7 & C8 & C9 & C10 & C11 & C12 & C13 & \begin{tabular}{|l|} 
C14 \\
\end{tabular} & \begin{tabular}{|l|} 
C15 \\
\end{tabular} & C16 & \begin{tabular}{|l|} 
C17 \\
\end{tabular} & 18 \\
\hline 01 & 122.25 & 10 & 7.49 & 1.8375 & 75.23 & 1.73 & 99.65 & 5 & 10 & 11 & 11 & 25 & \begin{tabular}{|l|}
77.34 \\
\end{tabular} & 20 & 16 & 30 & 14 & 1 \\
\hline $\mathrm{O2}$ & 131.78 & 2.6 & 8.88 & 575 & \begin{tabular}{|l|}
73.89 \\
\end{tabular} & 50 & 121.35 & 5 & 10 & 13 & $\varepsilon$ & 21 & 5.12 & 20 & 17 & 28 & 20 & 10 \\
\hline $\mathbf{O 3}$ & 129.75 & 31 & 7.64 & 1.4425 & 60.58 & 1.88 & 101.93 & 6 & 6 & 8 & 10 & 21 & 64.97 & 19 & 18 & 35 & 17 & 6 \\
\hline O4 & \begin{tabular}{|l|}
107.75 \\
\end{tabular} & 9.4 & 6.685 & 2.2425 & 67.85 & 2.15 & 106.50 & 7 & 9 & 9 & 11 & 24 & 75.51 & 17 & 19 & 33 & 18 & 8 \\
\hline 05 & 133.36 & 2.8 & .685 & 1.4675 & 55.19 & 2.19 & 111.07 & 6 & 8 & 9 & 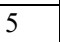 & 17 & \begin{tabular}{|l|}
70.65 \\
\end{tabular} & 10 & 6 & 52 & 13 & 7 \\
\hline O6 & 131 & 4.6 & 9.05 & 1.605 & 79.41 & 2.32 & 124.78 & 5 & s & 11 & c & 19 & 39.84 & 13 & 13 & 29 & 19 & 10 \\
\hline O7 & 112.09 & $\overline{9.6}$ & 7.38 & 1.6525 & 52.8 & 1.76 & 88.22 & 4 & 9 & 11 & 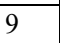 & 16 & 62.57 & $T^{2}$ & 14 & 40 & 17 & 6 \\
\hline O8 & 131.04 & 27.2 & 9.65 & 2.4375 & 79.11 & 2.51 & 112.21 & 5 & 10 & 10 & $\varepsilon$ & 22 & 62.82 & 19 & 17 & 36 & 21 & 10 \\
\hline O9 & 128.66 & 3 & 9.19 & 1.09 & 74 & 2.32 & 104.22 & 6 & 5 & 10 & 5 & 14 & 64.33 & 16 & 13 & 40 & 16 & 7 \\
\hline O10 & 148.39 & 33.6 & \begin{tabular}{|l|l|}
12.88 \\
\end{tabular} & 1.325 & 54.64 & 1.78 & 95.08 & 4 & 6 & 11 & 11 & 21 & 62.19 & 12 & 14 & 54 & 18 & 14 \\
\hline 011 & 13.94 & 22.2 & 9.015 & 1.2925 & 66.24 & 2.05 & 98.50 & 4 & 0 & 10 & 10 & 22 & 68.19 & 16 & 17 & 41 & 19 & 11 \\
\hline 012 & 132.66 & 2.4 & \begin{tabular}{|l|}
11.485 \\
\end{tabular} & 1.2925 & 74.39 & 2.05 & 116.78 & 4 & 8 & 12 & $\varepsilon$ & 18 & 63.45 & 16 & 16 & 45 & 15 & 13 \\
\hline 013 & 129.15 & 31.8 & 7.915 & 1.38 & 72.89 & 2.58 & 114.50 & 8 & 8 & 10 & 3 & 19 & 52.91 & 15 & 10 & 46 & 17 & 3 \\
\hline 014 & 22.38 & 0.2 & 9.97 & 2.33 & 71.42 & 1.97 & 111.07 & 6 & 9 & 8 & 9 & 26 & 65.78 & 17 & 19 & 27 & 16 & 10 \\
\hline O15 & 127.18 & 26 & 8.175 & 1.7225 & 52.68 & 1.68 & 114.50 & 7 & 10 & 12 & 2 & 23 & \begin{tabular}{|l|}
73.49 \\
\end{tabular} & 18 & 12 & 34 & 12 & 0 \\
\hline 016 & 29.57 & 16 & 8.33 & 1.3975 & 71.12 & 2.02 & 121.35 & 8 & 10 & 6 & 13 & 23 & 58.90 & 19 & 15 & 25 & 18 & 6 \\
\hline 017 & 159.65 & 29.6 & 11.97 & 1.805 & 92.6 & 1.64 & 97.36 & 3 & 9 & 7 & 7 & 20 & 54.93 & 15 & 19 & 46 & 21 & 16 \\
\hline 018 & 25.96 & 20.8 & 8.47 & 1.255 & 54.84 & 1.94 & 109.93 & 5 & 9 & 7 & 7 & 21 & 56.25 & 16 & 14 & 39 & 15 & 8 \\
\hline O19 & \begin{tabular}{|l|}
136.89 \\
\end{tabular} & 31 & 8.835 & 1.3275 & 74.31 & 1.92 & \begin{tabular}{|l|}
119.07 \\
\end{tabular} & 6 & 8 & 12 & 6 & 21 & 56.51 & 20 & 19 & 24 & 17 & 3 \\
\hline $\mathbf{O 2 0}$ & 24.87 & 26.4 & 7.31 & 1.38 & 64.97 & 1.78 & 90.51 & 3 & 6 & 8 & 10 & 19 & 60.67 & 14 & 11 & 45 & 15 & 7 \\
\hline O21 & 114.44 & 19.8 & 8.68 & 1.26 & 75.1 & 2.40 & 124.78 & 7 & 7 & 8 & 7 & 20 & 68.50 & 16 & 16 & 53 & 16 & 17 \\
\hline O22 & 122.25 & 10 & \begin{tabular}{|l}
7.49 \\
\end{tabular} & 1.8375 & 75.23 & 1.73 & 99.65 & 5 & 10 & 11 & 11 & 25 & 77.34 & 20 & 16 & 30 & 14 & 1 \\
\hline $\mathbf{O 2 3}$ & 131.78 & 22.6 & 8.88 & 1.575 & \begin{tabular}{|l|}
73.89 \\
\end{tabular} & 1.50 & 121.35 & 5 & 10 & 13 & 8 & 21 & 55.12 & 20 & 17 & 28 & 20 & 10 \\
\hline O24 & 129.75 & 31 & \begin{tabular}{|l}
7.64 \\
\end{tabular} & 1.4425 & 60.58 & 1.88 & 101.93 & 6 & 6 & 8 & 10 & 21 & 64.97 & 19 & 18 & 35 & 17 & 6 \\
\hline 025 & 107.75 & 29.4 & 6.685 & 2.2425 & 67.85 & 2.15 & 106.50 & 7 & 9 & 9 & 11 & 24 & 75.51 & 17 & 19 & 33 & 18 & 8 \\
\hline O26 & 133.36 & 42.8 & 7.685 & 1.4675 & 55.19 & 2.19 & 111.07 & 6 & 8 & 9 & 5 & 17 & 70.65 & 6 & 6 & 52 & 13 & 7 \\
\hline $\mathbf{O 2 7}$ & 131 & 14.6 & 9.05 & 1.605 & 79.41 & 2.32 & 124.78 & 5 & 9 & 11 & 6 & 19 & 39.84 & 13 & 13 & 29 & 19 & 10 \\
\hline $\mathbf{O 2 8}$ & 112.09 & 19.6 & 7.38 & 1.6525 & 52.8 & 1.76 & 88.22 & 4 & 9 & 11 & 9 & 16 & 62.57 & 9 & 14 & 40 & 17 & 6 \\
\hline O29 & 131.04 & 27.2 & 9.65 & 2.4375 & 79.11 & 2.51 & 112.21 & 5 & 10 & 10 & 8 & 22 & \begin{tabular}{|l|}
62.82 \\
\end{tabular} & 19 & 17 & 36 & 21 & 10 \\
\hline O30 & 128.66 & 23 & 9.19 & 1.09 & 74 & 2.32 & 104.22 & 6 & 5 & 10 & 5 & 14 & 64.33 & 16 & 13 & 40 & 16 & 7 \\
\hline O31 & 148.39 & 33.6 & 12.88 & 1.325 & 54.64 & 1.78 & 95.08 & 4 & 6 & 11 & 11 & 21 & 62.19 & 12 & 14 & 54 & 18 & 14 \\
\hline O32 & 113.94 & 22.2 & 9.015 & 1.2925 & 66.24 & 2.05 & 98.50 & 4 & 6 & 10 & 10 & 22 & 68.19 & 16 & 17 & 41 & 19 & 11 \\
\hline $\mathbf{O 3 3}$ & 132.66 & 32.4 & 11.485 & 1.2925 & 74.39 & 2.05 & \begin{tabular}{|l|l}
116.78 \\
\end{tabular} & 4 & 8 & 12 & 8 & 18 & \begin{tabular}{|l|}
63.45 \\
\end{tabular} & \begin{tabular}{|l|l}
16 \\
\end{tabular} & 16 & 45 & 15 & 13 \\
\hline
\end{tabular}

Table 1. Results from the tests (Criteria: C1-C18) of the first control group of 33 male students attending basketball (Objects: O1-O33). 


\begin{tabular}{|c|c|c|c|c|c|c|c|c|c|c|c|c|c|c|c|c|c|c|}
\hline & $\mathrm{C1}$ & $\mathrm{C} 2$ & $\mathrm{C} 3$ & 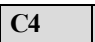 & C5 & 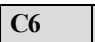 & 7 & 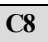 & 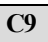 & 10 & 11 & 12 & 13 & C14 & C15 & 16 & 17 & 18 \\
\hline 01 & 06.02 & \begin{tabular}{|l|}
21.2 \\
\end{tabular} & .51 & 032 & 9.1 & 2.399 & 1.07 & 6 & 10 & 9 & 10 & 24 & 2.285 & 19 & 18 & 206.02 & 21.2 & 1.51 \\
\hline $\mathbf{O 2}$ & 2.44 & \begin{tabular}{|l|}
26.4 \\
\end{tabular} & 925 & 185 & 66.81 & 3.219 & 1.35 & 6 & & & & & .365 & 16 & 2 & 2.44 & 6.4 & 925 \\
\hline $\mathbf{0 3}$ & 8.16 & .4 & 105 & 37 & 12 & 3.56 & .503 & 7 & & 10 & 12 & 23 & 2.31 & 15 & 14 & 8.16 & \begin{tabular}{|l|}
33.4 \\
\end{tabular} & 2.105 \\
\hline O4 & 9.89 & \begin{tabular}{|l|}
28.2 \\
\end{tabular} & 755 & $\mid 1.0125$ & .94 & 2.34 & .791 & 6 & & & 10 & 23 & 5.53 & 19 & 6 & 9.89 & 28.2 & 9.755 \\
\hline 05 & 7.09 & 1.4 & 995 & \begin{tabular}{|l|}
1.1775 \\
\end{tabular} & .02 & 2.194 & 105.35 & 5 & 6 & 11 & 4 & 25 & 1.085 & 20 & 19 & 7.09 & \begin{tabular}{|l|}
34.4 \\
\end{tabular} & 9.995 \\
\hline O6 & 71.47 & 20 & 13.25 & 1.22 & 79.08 & 2.803 & 103.07 & 5 & 10 & 11 & & 17 & 55.18 & 11 & 11 & 71.47 & 20 & 13.25 \\
\hline 07 & 2.88 & \begin{tabular}{|l|}
19.8 \\
\end{tabular} & 945 & \begin{tabular}{|l|}
1.2075 \\
\end{tabular} & 16.09 & \begin{tabular}{|l|}
2.216 \\
\end{tabular} & 116.78 & 6 & 6 & 13 & 12 & 24 & 56.44 & 19 & 19 & 132.88 & \begin{tabular}{|l|}
19.8 \\
\end{tabular} & 8.945 \\
\hline 08 & 46.91 & \begin{tabular}{|l|}
21.4 \\
\end{tabular} & 7.34 & 1.16 & 73.15 & 1.685 & 101.93 & 3 & 10 & 10 & 11 & 26 & 65.595 & 15 & 15 & 146.91 & \begin{tabular}{|l|}
21.4 \\
\end{tabular} & 7.34 \\
\hline O9 & 46.9 & \begin{tabular}{|l|}
32.8 \\
\end{tabular} & .68 & 1.075 & .58 & \begin{tabular}{|l|l|}
1.770 \\
\end{tabular} & 112.21 & 6 & 5 & 9 & 5 & 21 & 6,06 & 18 & 13 & 146.9 & \begin{tabular}{|l|}
32.8 \\
\end{tabular} & 10.68 \\
\hline $\mathbf{O 1 0}$ & 9.76 & 23.2 & 12.76 & 1.3275 & 76.51 & 2.276 & 108.78 & 0 & 11 & & & 20 & 77.02 & 15 & 15 & 149.76 & \begin{tabular}{|l|}
23.2 \\
\end{tabular} & 12.76 \\
\hline 011 & 8.24 & \begin{tabular}{|l|}
19.6 \\
\end{tabular} & .08 & 1.4825 & 7.58 & 1.958 & 120.20 & 6 & 10 & 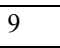 & & 18 & 8.495 & 16 & 14 & 118.24 & \begin{tabular}{|l|}
19.6 \\
\end{tabular} & 10.08 \\
\hline 012 & 4.34 & 22 & 10.275 & 1.225 & 73.12 & 2.125 & 117.92 & 6 & & & & 25 & 69.825 & 19 & 18 & 154.34 & 22 & 10.275 \\
\hline 013 & 3.42 & 20.2 & 0.24 & 0.975 & 82.43 & \begin{tabular}{|l|}
1.9901 \\
\end{tabular} & 113.3 & - & & & & 3 & 3.195 & 18 & 14 & 3.42 & 20.2 & 10.24 \\
\hline 014 & 63.36 & \begin{tabular}{|l|}
17.6 \\
\end{tabular} & 9.185 & \begin{tabular}{|l|}
1.6825 \\
\end{tabular} & 68.38 & 2.840 & 105.35 & 6 & 10 & 10 & 8 & 24 & 79.925 & 13 & 18 & 163.36 & \begin{tabular}{|l|}
17.6 \\
\end{tabular} & 9.185 \\
\hline 015 & 2.24 & \begin{tabular}{|l|}
21.4 \\
\end{tabular} & 99 & 1.275 & 73.48 & 2.006 & 109.92 & - & & 1 & & 27 & 69.635 & 14 & 14 & 12.24 & \begin{tabular}{|l|}
21.4 \\
\end{tabular} & 7.99 \\
\hline 016 & 15.65 & \begin{tabular}{|l|}
22.6 \\
\end{tabular} & 9.945 & 1.53 & 89.23 & 1.64 & \begin{tabular}{|l|l}
111.07 \\
\end{tabular} & 6 & 8 & 10 & 7 & 24 & 50.125 & 7 & 13 & 115.65 & \begin{tabular}{|l|}
22.6 \\
\end{tabular} & 9.945 \\
\hline 017 & 8.94 & 7.8 & 3.245 & 17 & 83.17 & 2.343 & \begin{tabular}{|l|}
111.07 \\
\end{tabular} & 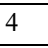 & - & & 10 & 27 & 73.045 & 20 & 17 & 198.94 & \begin{tabular}{|l|}
17.8 \\
\end{tabular} & 13.245 \\
\hline 018 & 28.76 & \begin{tabular}{|l|}
15.4 \\
\end{tabular} & 8.71 & \begin{tabular}{|l|}
1.1825 \\
\end{tabular} & 59.01 & 2.44 & 124.7 & 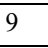 & 10 & $\varepsilon$ & 10 & 20 & 59.72 & 15 & 13 & 128.76 & \begin{tabular}{|l|}
15.4 \\
\end{tabular} & 8.71 \\
\hline O19 & 3.41 & 21 & 8.675 & 1.23 & 67.02 & 1.88 & 115.639 & 3 & 10 & 8 & & 24 & 70.325 & 15 & 17 & 133.41 & 21 & 8.675 \\
\hline $\mathbf{O 2 0}$ & 78.07 & \begin{tabular}{|l|}
20.8 \\
\end{tabular} & 9.685 & 0.91 & 84.98 & 2.756 & 104.21 & 6 & 5 & 10 & 12 & 26 & 77.465 & 11 & 7 & 178.07 & \begin{tabular}{|l|}
20.8 \\
\end{tabular} & 9.685 \\
\hline 021 & 6.11 & 10.8 & 51 & 1.025 & 64.3 & 2.26 & 112.2 & 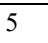 & 8 & 10 & 1 & 23 & 72.79 & 19 & 10 & 136.11 & \begin{tabular}{|l|}
10.8 \\
\end{tabular} & 8.51 \\
\hline O22 & 28.37 & \begin{tabular}{|l|}
27.2 \\
\end{tabular} & 7.925 & \begin{tabular}{|l|}
1.3675 \\
\end{tabular} & 57.5 & \begin{tabular}{|l|}
1.60 \\
\end{tabular} & 100.78 & 4 & 9 & 12 & 8 & \begin{tabular}{|l|}
13 \\
\end{tabular} & 61.36 & 15 & 13 & 128.37 & \begin{tabular}{|l|}
27.2 \\
\end{tabular} & 7.925 \\
\hline $\mathbf{O 2 3}$ & 177.05 & \begin{tabular}{|l|}
20.4 \\
\end{tabular} & 10.04 & 1.3325 & 60.79 & 3.162 & 100.78 & 0 & 6 & & 9 & 18 & 61.175 & 20 & 17 & 177.05 & \begin{tabular}{|l|}
20.4 \\
\end{tabular} & 10.04 \\
\hline 024 & 94.75 & \begin{tabular}{|l|}
18.6 \\
\end{tabular} & 2.915 & 0.865 & \begin{tabular}{|l|}
123.61 \\
\end{tabular} & 3.26 & 111.070 & 6 & 3 & 9 & 11 & 20 & 63.13 & 19 & 18 & 194.75 & \begin{tabular}{|l|}
18.6 \\
\end{tabular} & 12.915 \\
\hline $\mathbf{O 2 5}$ & 208.81 & \begin{tabular}{|l|}
20.4 \\
\end{tabular} & 9.495 & 1.7625 & 77.43 & 1.894 & 104.21 & 6 & 10 & 13 & 6 & 22 & 65.655 & 16 & 16 & 208.81 & \begin{tabular}{|l|}
20.4 \\
\end{tabular} & 9.495 \\
\hline 026 & 207.05 & \begin{tabular}{|l|}
15.6 \\
\end{tabular} & 8.605 & 1.34 & 74.68 & 2.686 & 113.35 & 6 & 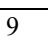 & 11 & 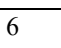 & 21 & 52.085 & 19 & 17 & 207.05 & \begin{tabular}{|l|}
15.6 \\
\end{tabular} & 8.605 \\
\hline $\mathbf{O 2 7}$ & 195.21 & 26 & 12.98 & 0.98 & 73.28 & 1.84 & 99.645 & 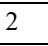 & 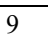 & 4 & 7 & 22 & 62.06 & 15 & 16 & 195.21 & 26 & 12.98 \\
\hline O28 & 160.46 & \begin{tabular}{|l|}
36.4 \\
\end{tabular} & 9.515 & \begin{tabular}{|l|}
1.3625 \\
\end{tabular} & 74.28 & 2.54 & 96.21 & 5 & 7 & 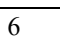 & 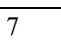 & 22 & 52.65 & 4 & 10 & 160.46 & \begin{tabular}{|l|}
36.4 \\
\end{tabular} & 9.515 \\
\hline O29 & 163.91 & \begin{tabular}{|l|}
12.6 \\
\end{tabular} & 9.205 & 1.305 & 85.91 & 3.30 & 96.21 & 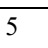 & 0 & 4 & 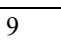 & 18 & 60.92 & 13 & 10 & 163.91 & \begin{tabular}{|l|}
12.6 \\
\end{tabular} & 9.205 \\
\hline $\mathbf{0 3 0}$ & \begin{tabular}{|l|}
148.1 \\
\end{tabular} & \begin{tabular}{|l|}
48.4 \\
\end{tabular} & 8.235 & \begin{tabular}{|l|}
1.4075 \\
\end{tabular} & 69.76 & 2.676 & 111.07 & 6 & 10 & 8 & 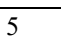 & 23 & 59.03 & 16 & 16 & 148.1 & \begin{tabular}{|l|}
48.4 \\
\end{tabular} & 8.235 \\
\hline O31 & 124.94 & 16 & 8.215 & \begin{tabular}{|l|}
1.4375 \\
\end{tabular} & 70.35 & \begin{tabular}{|l|}
1.97 \\
\end{tabular} & 105.35 & 5 & 5 & 10 & 12 & 25 & 77.08 & 20 & 18 & 124.94 & 16 & 8.215 \\
\hline $\mathbf{O 3 2}$ & 159.12 & \begin{tabular}{|l|}
19.6 \\
\end{tabular} & \begin{tabular}{|l|}
12.085 \\
\end{tabular} & \begin{tabular}{|l|}
1.6275 \\
\end{tabular} & 88.51 & 2.235 & 129.34 & 6 & 10 & 11 & 10 & 23 & 59.47 & 19 & 16 & 159.12 & \begin{tabular}{|l|}
19.6 \\
\end{tabular} & 12.085 \\
\hline $\mathbf{O 3 3}$ & 206.02 & \begin{tabular}{|l|}
21.2 \\
\end{tabular} & 11.51 & 1.0325 & 79.1 & 2.399 & 111.07 & 6 & 10 & 9 & 10 & 24 & 72.285 & 19 & 18 & 206.02 & 21.2 & 11.51 \\
\hline
\end{tabular}

Table 2. Results from the tests (Criteria: C1-C18) of the first control group of 33 male students attending table tennis (Objects: O1-O33).

\section{Application of the InterCriteria Analysis}

The method of InterCriteria Analysis is based on intuitionistic fuzzy sets, thus rendering account of the effects of uncertainty. Originally, ICrA was been proposed in [2], and various aspects of its theoretical investigation are given in papers $[1,3,4]$.

For this aim, we use the developed software for InterCriteria Analysis [10] (freely available online at: http://intercriteria.net/software) and feed it with the data from the two control groups of students. The complete resultant tables with InterCriteria intuitionistic fuzzy membership pairs are given on Table 3 and Table 4 .

While not strictly positive consonances are detected, we see that the strongest available ones, i.e., those with smallest distance from the intuitionistic fuzzy truth $\langle 1,0\rangle$, are those detected between the C18 (Anxiety) - C16 (Neuroticism), C18 (Anxiety) - C3 (Agility) and C5 (Operational thinking) - C6 (Skillfulness). These pairs are formed between logically related qualities. All other pairs score intuitionistic fuzzy dissonance values and are located in to the respective zone of the IF triangle showed on Figure 1. 


\begin{tabular}{|c|c|c|c|c|c|c|c|c|c|c|c|c|c|c|c|c|c|c|}
\hline & $\mathrm{Cl}$ & $\mathrm{C} 2$ & $\mathrm{C} 3$ & $\mathrm{C} 4$ & C5 & C6 & $\mathrm{C} 7$ & C8 & C9 & $\mathrm{C} 10$ & C11 & $\mathrm{C} 12$ & $\mathrm{C} 13$ & $\mathrm{Cl} 4$ & $\mathrm{C} 15$ & $\mathrm{C} 16$ & $\mathrm{C} 17$ & $\mathrm{C} 18$ \\
\hline $\mathrm{Cl}$ & & 0.5508 & 0.6292 & 0.4973 & 0.5437 & 0.4955 & 0.4866 & 0.3173 & 0.3957 & 0.5472 & 0.3244 & 0.3832 & 0.344 & 0.4385 & 0.385 & 0.5276 & 0.5152 & 0.5526 \\
\hline $\mathrm{C} 2$ & 0.5508 & & 0.59 & 0.5134 & 0.4278 & 0.4866 & 0.4759 & 0.4777 & 0.3298 & 0.4742 & 0.3939 & 0.4385 & 0.5579 & 0.4029 & 0.5062 & 0.5312 & 0.4795 & 0.4385 \\
\hline $\mathrm{C} 3$ & 0.6292 & 0.59 & 1 & 0.5045 & 0.6007 & 0.5258 & 0.5829 & 0.4011 & 0.41 & 0.4866 & 0.3779 & 0.4742 & 0.3832 & 0.4617 & 0.5294 & 0.508 & 0.5526 & 0.6203 \\
\hline $\mathrm{C} 4$ & 0.4973 & 0.5134 & 0.5045 & & 0.4706 & 0.4938 & 0.4902 & 0.4242 & 0.5365 & 0.3993 & 0.5223 & 0.5847 & 0.4831 & 0.492 & 0.4884 & 0.4474 & 0.4759 & 0.4706 \\
\hline C5 & 0.5437 & 0.4278 & 0.6007 & 0.4706 & & 0.6132 & 0.5651 & 0.4171 & 0.4492 & 0.3815 & 0.4225 & 0.5419 & 0.4439 & 0.5241 & 0.4635 & 0.4563 & 0.4563 & 0.5276 \\
\hline C6 & 0.4955 & 0.4866 & 0.5258 & 0.4938 & 0.6132 & 1 & 0.5775 & 0.4866 & 0.4082 & 0.3636 & 0.3672 & 0.3957 & 0.4777 & 0.4225 & 0.344 & 0.5936 & 0.4724 & 0.5847 \\
\hline C7 & 0.4866 & 0.4759 & 0.5829 & 0.4902 & 0.5651 & 0.5775 & & 0.5811 & 0.4955 & 0.4225 & 0.3262 & 0.4848 & 0.3975 & 0.5704 & 0.467 & 0.3512 & 0.4367 & 0.4617 \\
\hline C8 & 0.3173 & 0.4777 & 0.4011 & 0.4242 & 0.4171 & 0.4866 & 0.5811 & & 0.4278 & 0.3619 & 0.3298 & 0.4545 & 0.4955 & 0.4171 & 0.4082 & 0.2816 & 0.3529 & 0.2353 \\
\hline C9 & 0.3957 & 0.3298 & 0.41 & 0.5365 & 0.4492 & 0.4082 & 0.4955 & 0.4278 & & 0.4296 & 0.3922 & 0.4474 & 0.3743 & 0.4456 & 0.426 & 0.2852 & 0.4332 & 0.4029 \\
\hline $\mathrm{C} 10$ & 0.5472 & 0.4742 & 0.4866 & 0.3993 & 0.3815 & 0.3636 & 0.4225 & 0.3619 & 0.4296 & & 0.3155 & 0.3547 & 0.4706 & 0.3761 & 0.4296 & 0.3547 & 0.4403 & 0.3583 \\
\hline C11 & 0.3244 & 0.3939 & 0.3779 & 0.5223 & 0.4225 & 0.3672 & 0.3262 & 0.3298 & 0.3922 & 0.3155 & 1 & 0.6078 & 0.533 & 0.4439 & 0.5027 & 0.3797 & 0.3886 & 0.4403 \\
\hline $\mathrm{C} 12$ & 0.3832 & 0.4385 & 0.4742 & 0.5847 & 0.5419 & 0.3957 & 0.4848 & 0.4545 & 0.4474 & 0.3547 & 0.6078 & & 0.5633 & 0.5758 & 0.5686 & 0.2941 & 0.3583 & 0.3547 \\
\hline $\mathrm{C} 13$ & 0.344 & 0.5579 & 0.3832 & 0.4831 & 0.4439 & 0.4777 & 0.3975 & 0.4955 & 0.3743 & 0.4706 & 0.533 & 0.5633 & & 0.4474 & 0.5223 & 0.4831 & 0.3298 & 0.3957 \\
\hline $\mathrm{C} 14$ & 0.4385 & 0.4029 & 0.4617 & 0.492 & 0.5241 & 0.4225 & 0.5704 & 0.4171 & 0.4456 & 0.3761 & 0.4439 & 0.5758 & 0.4474 & & 0.59 & 0.2656 & 0.4617 & 0.3494 \\
\hline $\mathrm{C} 15$ & 0.385 & 0.5062 & 0.5294 & 0.4884 & 0.4635 & 0.344 & 0.467 & 0.4082 & 0.426 & 0.4296 & 0.5027 & 0.5686 & 0.5223 & 0.59 & & 0.2906 & 0.5312 & 0.4528 \\
\hline C16 & 0.5276 & 0.5312 & 0.508 & 0.4474 & 0.4563 & 0.5936 & 0.3512 & 0.2816 & 0.2852 & 0.3547 & 0.3797 & 0.2941 & 0.4831 & 0.2656 & 0.2906 & 1 & 0.4403 & 0.6435 \\
\hline C17 & 0.5152 & 0.4795 & 0.5526 & 0.4759 & 0.4563 & 0.4724 & 0.4367 & 0.3529 & 0.4332 & 0.4403 & 0.3886 & 0.3583 & 0.3298 & 0.4617 & 0.5312 & 0.4403 & & 0.5579 \\
\hline $\mathrm{C} 18$ & 0.5526 & 0.4385 & 0.6203 & 0.4706 & 0.5276 & 0.5847 & 0.4617 & 0.2353 & $\begin{array}{l}0.4029 \\
\end{array}$ & 0.3583 & 0.4403 & 0.3547 & 0.3957 & 0.3494 & 0.4528 & 0.6435 & 0.5579 & \\
\hline
\end{tabular}

Table 3. Results of the application of the InterCriteria Analysis on the aggregated data from Table 1, IF membership parts

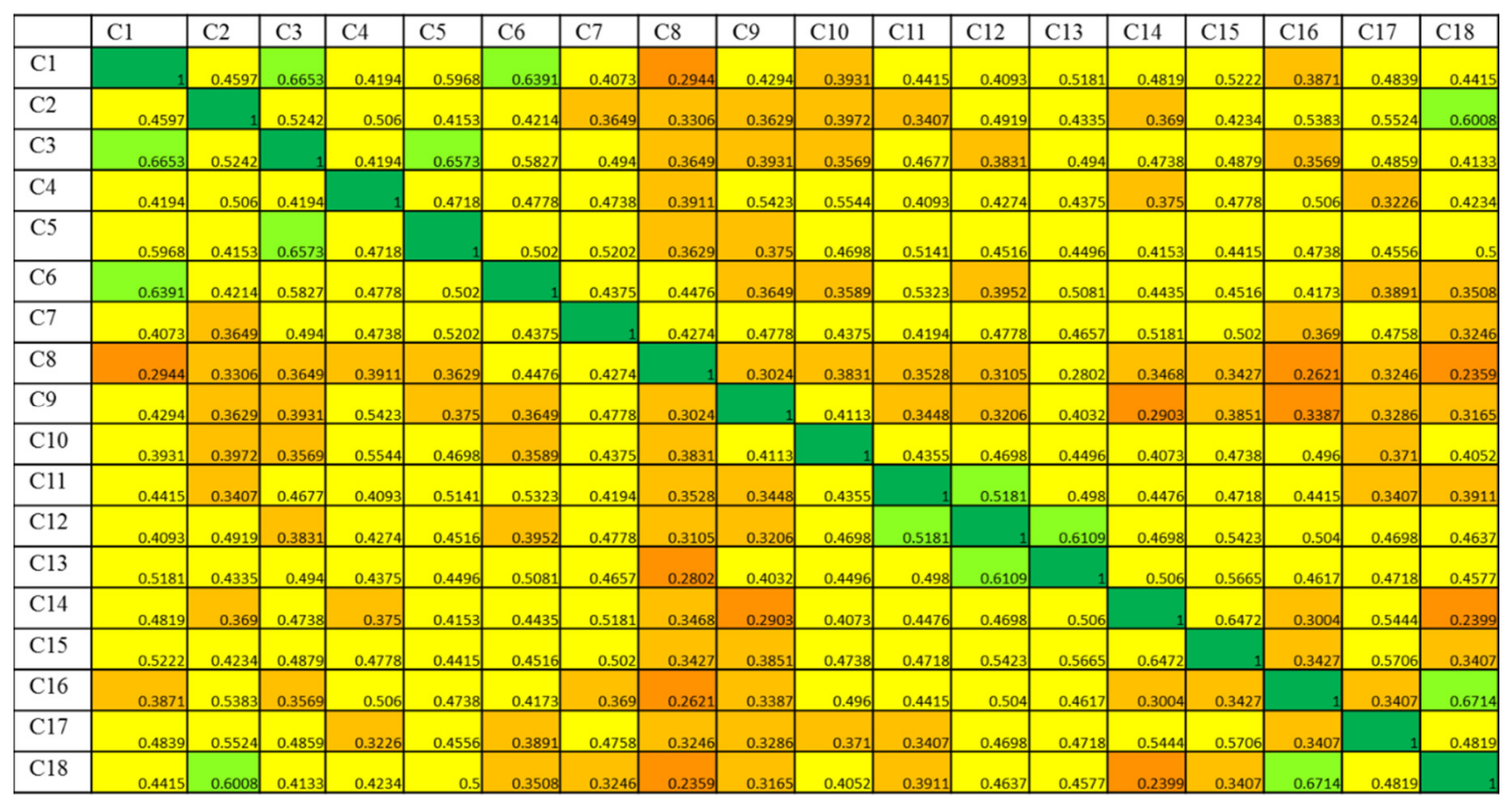

Table 4. Results of the application of the InterCriteria Analysis on the aggregated data from Table 2, IF membership parts

The results represented on Table 4 from the application of the InterCriteria Analysis on the aggregated data from the second control group of students are showing similar behaviour. The only difference is detected between the consonance values of C1 (Endurance) - C5 (Operational thinking). The graphical representation of the results is presented on Figure 2. 


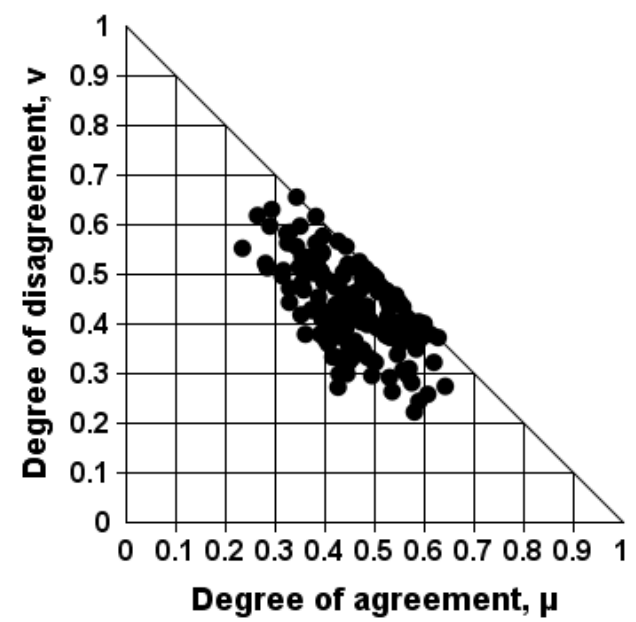

Figure 1. InterCriteria pairs between the 18 criterions (Table 3 ), as plotted as points (black) on the intuitionistic fuzzy interpretational triangle

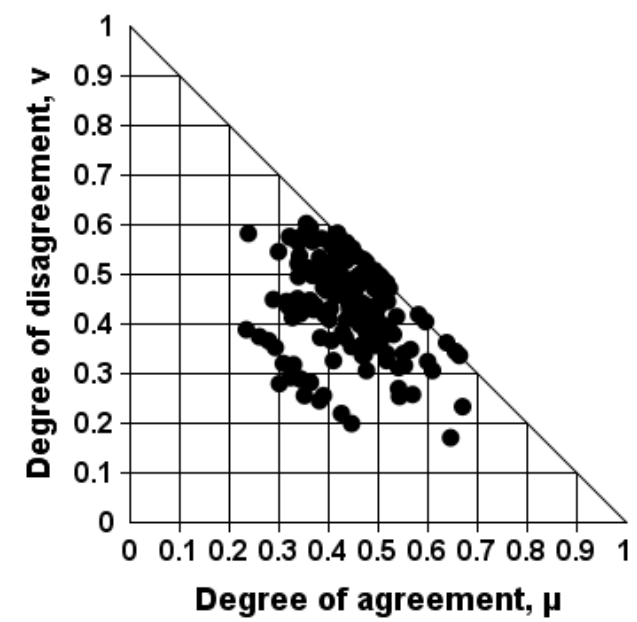

Figure 2. InterCriteria pairs between the 18 criterions (Table 4), as plotted as points (black) on the intuitionistic fuzzy interpretational triangle

\section{Conclusions}

The study describes a new method of processing data obtained from university students practicing sports activities and the results obtained from the application of ICrA shows that the chosen tests in the test battery are very well combined to evaluate the initial level of intellectual and physical development of the students. Naturally, given the relatively small size of the considered data it is not possible to claim with absolute certainty that our interpretations are doubtlessly valid but they provide a starting point for further investigations.

\section{Acknowledgements}

The first author is grateful for the support provided under Grant No. KP-06-N-22/1/2018 "Theoretical research and applications of InterCriteria Analysis" funded by the National Science Fund of Bulgaria.

\section{References}

[1] Atanassov, K., Atanassova, V. \& Gluhchev, G. (2015). InterCriteria Analysis: Ideas and problems, Notes on Intuitionistic Fuzzy Sets, 21 (1), 81-88.

[2] Atanassov, K., Mavrov, D., \& Atanassova, V. (2014). Intercriteria Decision Making: A New Approach for Multicriteria Decision Making, Based on Index Matrices and Intuitionistic Fuzzy Sets. Issues in Intuitionistic Fuzzy Sets and Generalized Nets, 11, $1-8$.

[3] Atanassov, K., Szmidt, E., Kacprzyk, J., \& Atanassova, V. (2017). An approach to a constructive simplification of multiagent multicriteria decision making problems via 
intercriteria analysis. Comptes rendus de l'Academie bulgare des Sciences, 70 (8), $1147-1156$.

[4] Atanassova, V. (2015). Interpretation in the Intuitionistic Fuzzy Triangle of the Results, Obtained by the InterCriteria Analysis, $16^{\text {th }}$ World Congress of the International Fuzzy Systems Association (IFSA), $9^{\text {th }}$ Conference of the European Society for Fuzzy Logic and Technology (EUSFLAT), 30.06 - 03.07.2015, Gijon, Spain, 1369-1374.

[5] Bailey, R., Hillman, C., Arent, S., \& Petitpas, A. (2013). Physical activity: An underestimated investment in human capital, J Phys ActHealth 2013 (10), 289-308.

[6] Chacón-Cuberos, R., Zurita-Ortega, F., et.al. (2018). Relationship between healthy habits and perceived motivational climate in sport among university students: A structural equation model. Sustainability. 10:938. doi: 10.3390/su10040938.

[7] Dishman, R. K., \& Dunn, A. L. (1988). Exercise adherence in children and youth: implications for adulthood. In: Dishman, $R$. K. (Ed). Exercise adherence: its impact on public health, Champaign, IL: Human Kinetics, 155-200.

[8] Haase, A., Steptoe, A., Sallis, J. F., \& Wardle, J. (2004). Leisure-time physical activity in university students from 23 countries: associations with health beliefs, risk awareness, and national economic development, Prev Med, 39, 182-190. doi: 10.1016/j.ypmed.2004. 01.028 .

[9] Jacobsen, W.C., \& Forste, R. (2011). The wired generation: Academic and social outcomes of electronic media use among university students, Cyberpsychol. Behav. Soc. Network., 14, 275-280. doi: 10.1089/cyber.2010.0135.

[10] Ikonomov, N., Vassilev, P., \& Roeva, O. (2018). ICrAData - Software for InterCriteria Analysis, Int J Bioautomation, 22 (1), 1-10.

[11] Keating, X. D., Guan, J., Pinero, J. C., \& Bridges, D. M. (2005). A metaanalysis of college students' physical activity behaviors. $J$ Am Coll Health, 54, 116-126. doi: 10.3200/JACH.54.2.116-126. 